\title{
Caracterização do tráfego de rede no domínio de controle do OpenStack produzido pela mudança de estado de máquinas virtuais
}

\author{
Adnei W. Donatti ${ }^{1}$, Guilherme P. Koslovski ${ }^{1}$, Charles C. Miers ${ }^{1}$ \\ ${ }^{1}$ Programa de Pós-Graduação em Computação Aplicada (PPGCA) \\ Departamento de Ciência da Computação - Universidade do Estado de Santa Catarina
}

\author{
adnei.donatti@edu.udesc.br, \\ \{guilherme.koslovski, charles.miers\}@udesc.br
}

\begin{abstract}
Resumo. O OpenStack distribui o tráfego de rede entre diversas interfaces e redes virtualizadas, que conectam servidores com serviços de nuvem, divididos em domínio de controle, público e convidado. O tráfego de controle do OpenStack ocorre através de requisições Rest oriundas de serviços OpenStack e RabbitMQ. O presente trabalho tem como objetivo caracterizar o tráfego de rede no domínio de controle do OpenStack produzido pela mudança de estado da máquina virtual (MV) definindo quanto é trafegado usando requisições REST e classificando o tráfego agregado no RabbitMQ.
\end{abstract}

\section{Introdução e Motivação}

A criação e gerenciamento de nuvens computacionais Infrastructure as a Service (IaaS), de um modo geral, é realizada através de um conjunto de ferramentas que aliam técnicas de sistemas distribuídos à virtualização, de modo a criar um ambiente no qual é possível fornecer recursos computacionais [OpenStack, 2019b]. O OpenStack é considerado um sistema operacional para nuvens, que controla um amplo grupo de recursos de computação, armazenamento e rede por todo o data center.

Analisando os componentes internos e funcionamento do OpenStack, nota-se que existe considerável necessidade de comunicação entre serviços. Por tratar-se de um sistema distribuído, é necessário coordenar e atualizar informações entre os serviços da nuvem. Neste sentido, o OpenStack trabalha com filas de mensagens gerenciadas pelo Advanced Message Queuing Protocol (AMQP), que pode ser implementado a partir de diversos serviços de mensageria, e.g., RabbitMQ (http://rabbitmq.com), Qpid (http: //qpid.apache.org), ZeroMQ (http://zeromq.org). Comumente, usa-se o RabbitMQ, devido ao suporte da maioria das aplicações [OpenStack, 2019a]. Dessa forma, para fins de completude da caracterização de tráfego administrativo do OpenStack, nota-se a necessidade da caracterização de chamadas Remote Procedure Call (RPC), que são gerenciadas pelo RabbitMQ, durante o ciclo de vida induzido das instâncias. Tal caracterização auxilia na identificação dos serviços utilizados durante o cenário de execução dos experimentos.

O presente trabalho traz a proposta de análise e caracterização do tráfego da rede administrativa do OpenStack, a fim de identificar serviços e operações em nível de camada de rede que estão sendo executadas durante o uso da nuvem. Além disso, a caracterização/classificação do tráfego interno do OpenStack ajuda nas configurações 
de redes, no sentido de melhor distribuir/configurar os recursos disponíveis (e.g., separar as redes de acordo com políticas de segurança, uso de rede, alocar/desalocar recursos de rede) a fim de ter ganhos em desempenho e segurança da nuvem. Foi definida uma implantação mínima do OpenStack Queens no CloudLab (https://cloudlab.us), plataforma para testes em nuvem que fornece ambiente isolado e de fácil acesso (Figura 1). Assim, faz-se a medição de tráfego de acordo com um ciclo de vida induzido das MVs, que atende às operações mais comuns em instâncias de MVs, criação, suspensão, reativação, parada e engavetamento (CREATE, SUSPEND, RESUME, STOP, SHELVE).

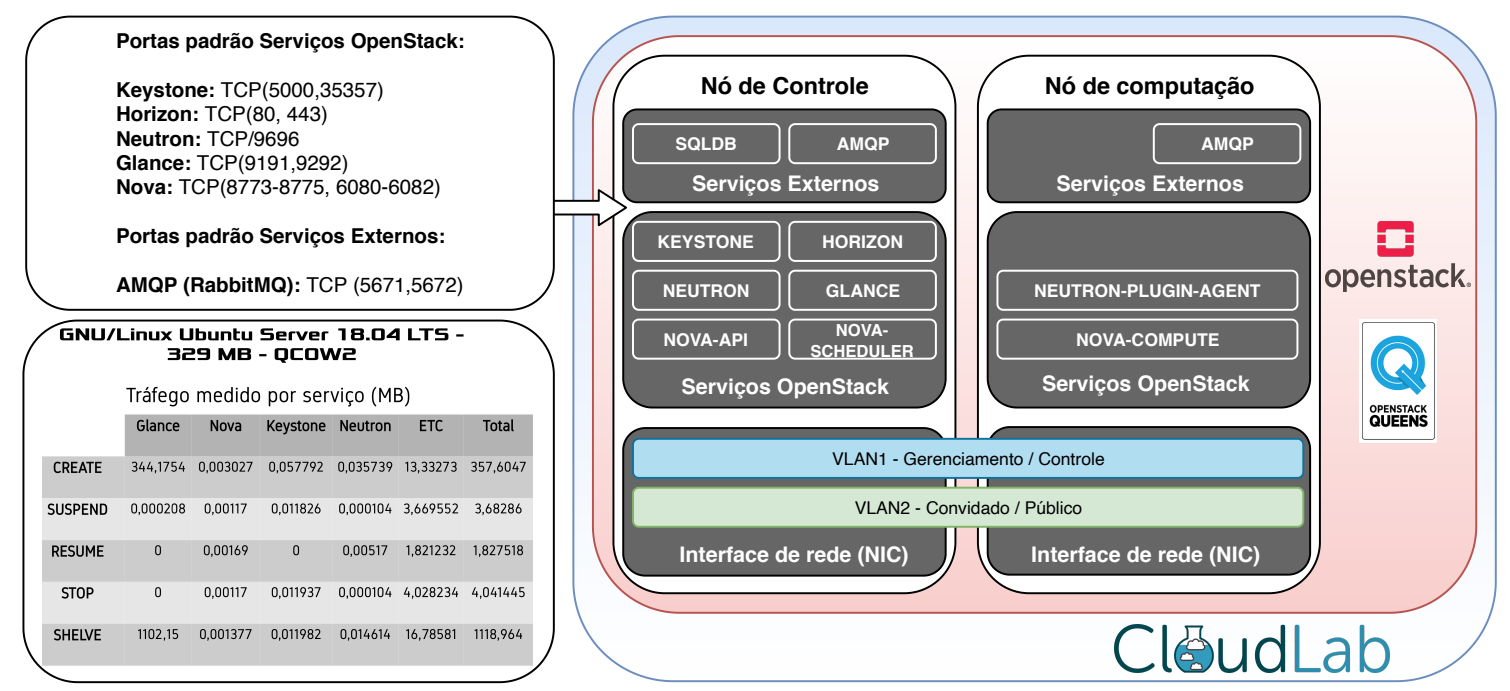

Figura 1. Configuração e resultados iniciais dos experimentos [Miers et al., 2019]

\section{Considerações e Trabalhos futuros}

Atualmente já está implementada e caracterizada a parte de tráfego de rede de controle dos Serviços OpenStack que empregam Rest usando as portas TCP (Figura 1). Contudo, essa análise inicial revelou que parte considerável do tráfego gerado nas operações é de RabbitMQ (ETC na tabela inclusa na Figura 1) que agrega todos os serviços em chamadas assíncronas usando protocolo RPC. Assim, está sendo desenvolvida uma ferramenta/método de monitoração que permita classificar as chamadas do RabbitMQ de acordo com o serviço do OpenStack que as solicitou (e.g., Nova e Glance).

Agradecimentos: Os autores agradecem o apoio do LabP2D/UDESC e da FAPESC.

\section{Referências}

Miers, C. C., Koslovski, G. P., Pillon, M. A., and Donatti, A. W. (2019). Análise do tráfego de máquinas virtuais na rede de controle de nuvens computacionais baseadas em OpenStack. In Livro de Minicursos da 17 a Escola Regional de Redes de Computadores, chapter 4, pages 1-19. Alegrete-RS, Brasil.

OpenStack (2019a). Message queue. https://docs.openstack.org/mitaka/install-guide-ubuntu/ environment-messaging.html. Acessado em 10 de janeiro 2020.

OpenStack (2019b). Vision for openstack clouds. https://governance.openstack.org/tc/reference/ technical-vision.html?_ga=2.75225810.1135708675.1574873893-711996610.1570721857. Acessado em 10 de janeiro 2020. 